\title{
Uso de la resonancia magnética y la tomografía computarizada en diagnóstico postmortem
}

\author{
The Use of Magnetic Resonance Imaging and Computed \\ Tomography Scans in Postmortem Diagnosis
}

\section{Uso da ressonância magnética e da tomografia computadorizada no diagnóstico pós-morte: revisão do assunto}

Andrea Paola Najar-Céspedes, Bt., MSc. *

Esteban de Jesús Fuentes-Martínez, Tnlgo. **

\begin{abstract}
Resumen
Introducción: Ante la naturaleza heterogénea, complejidad inherente de la actividad de peritaje en medicina legal, el perito (profesional de la medicina o áreas afines) debe hacer el mejor uso de las herramientas técnicas y tecnológicas de las cuales disponga. La imagenología, referente al conjunto de las técnicas que permiten obtener imágenes del cuerpo humano con fines clínicos o científicos, en cualquiera de sus técnicas, reviste una potente herramienta de apoyo estableciendo hechos o pruebas técnicas en el campo legal. Objetivo: Analizar el uso de la resonancia magnética y la tomografía computarizada en el diagnóstico postmortem. Metodología: Se realizó búsqueda de información en las bases de datos PubMed, Science Direct, Springer Journal y en el motor de búsqueda Google Académico, usando los términos "Tomografía Computarizada por Rayos X", "Espectroscopía de Resonancia Magnética", "Autopsia" y
\end{abstract}

\begin{abstract}
"Medicina Legal" publicados en el periodo 2008-2015. Resultados: La resonancia magnética es útil para el estudio detallado de tejidos blandos y órganos, mientras que la tomografía computarizada permite la identificación de fracturas, calcificaciones, implantes y traumas. Conclusiones: En los reportes hallados en la búsqueda bibliográfica, en cuanto al uso de la resonancia magnética nucleary la tomografía computarizada en casos postmortem, nombrados por la génesis del trauma, se halló correlación entre la utilización de la imagen y el correcto diagnóstico pericial en la autopsia. [Najar-Céspedes AP, FuentesMartínez EDJ. Uso de la resonancia magnética y la tomografía computarizada en diagnóstico postmortem. MedUNAB 2017; 20(2): 190-200].
\end{abstract}

Palabras clave: Diagnóstico por Imagen; Tomografía Computarizada por Rayos X; Espectroscopía de Resonancia Magnética; Autopsia; Medicina legal.

\footnotetext{
* Bacterióloga, magíster en Microbiología, Universidad Nacional de Colombia, docente investigador, Fundación Universitaria del Área Andina, Bogotá, Cundinamarca, Colombia.

** Tecnólogo en Radiología e Imágenes Diagnósticas, Fundación Universitaria del Área Andina, Asesor comercial Esmerald Mart SAS, Bogotá, Cundinamarca, Colombia.
} 


\section{Abstract}

Introduction: Given the heterogeneous nature, the inherent complexity to the activity of expertise in legal medicine, the expert (a professional in medicine or related areas) should make the best use of the technical and technological tools available for this. The imaging, which refers to the set of techniques that allow getting images of the human body for clinical or scientific purposes in any of its techniques, has a powerful support tool to establish facts or technical evidence in the legal field. Objective: To analyze the use of magnetic resonance imaging and computed tomography scan in postmortem diagnosis. Methodology: The search for information was carried out in the PubMed, Science Direct, Springer Journal databases and the Google Scholar search engine, by using the terms "X-ray Computed Tomography", "Magnetic Resonance Spectroscopy", "Autopsy", and "Legal Medicine" including articles published from 2008 to 2015. Results: A magnetic resonance imaging is useful for a detailed study of soft tissues and organs, while a computed tomography scan allows identifying fractures, calcifications, implants, and traumas. Conclusions: In the reports found in the literature search, regarding the use of nuclear magnetic resonance imaging and computed tomography scan in postmortem cases which were named after the genesis of the trauma, a correlation was found between the use of the image and the correct expert diagnosis at autopsy. [Najar-Céspedes AP, Fuentes-Martínez EDJ. The Use of Magnetic Resonance Imaging and Computed Tomography Scans in Postmortem Diagnosis. MedUNAB 2017; 20(2): 190-200].

Keywords: Diagnostic Imaging; Tomography, X-Ray Computed; Magnetic Resonance Spectroscopy; Autopsy; Forensic Medicine.

\section{Introducción}

La medicina legal o medicina forense, comprende la aplicación de la ciencia médica para apoyar técnica y científicamente a la administración de justicia. Como instituto, su ente rector en el caso colombiano es el Instituto Colombiano de Medicina Legal, organismo estatal compuesto por profesionales de las áreas médicas y afines, con el fin de emitir peritaciones que sirvan de pruebas técnicas en el esclarecimiento de los hechos(1).

Principalmente, la medicina legal se reconoce por su labor en el campo de la aplicación práctica del peritaje médicolegal; el cual cubre una amplia gama de pericias del orden psiquiátrico o psicológico, hasta aplicaciones con fines postmortem, en la determinación de la manera de muerte (Suicida, homicida o accidental).

Es cifra a resaltar que, el $2.5 \%$ de la mortalidad en el mundo es consecuencia de la violencia, en sus diferentes expresiones. En el 2012, a nivel mundial, se presentó una tasa de 6.7 homicidios por 100,000 habitantes. Sin embargo, la tasa más alta de homicidios ocurre en la región de las Américas, con un 28.5 por cada 100,000 habitantes(2). En Colombia, el informe FORENSIS 2015 menciona que, para el año 2017 , se reportaron 24,681 muertes violentas, dentro

\section{Resumo}

Introdução: Dada a natureza heterogênea, a complexidade inerente à experiência em medicina legal, o especialista (profissional médico ou áreas afins) deve fazer o melhor uso das ferramentas técnicas e tecnológicas disponíveis. A imagem, referente ao conjunto de técnicas que permitem imagens do corpo humano para fins clínicos ou científicos em qualquer um de seus métodos, é uma poderosa ferramenta de suporte que estabelece fatos ou testes técnicos no campo jurídico. Objetivo: Analisar o uso da ressonância magnética e da tomografia computadorizada no diagnóstico pós-morte. Metodologia: A pesquisa da informação foi realizada na base de dados da PubMed, da Science Direct, do Springer Journal e no motor de busca Google Scholar, usando os termos "tomografia computadorizada de raios-X", "Espectroscopia de Ressonância Magnética", "Autopsy "E" Medicina Legal ", incluindo artigos publicados no período 2008-2015. Resultados: MRI é útil para o estudo pormenorizado de tecidos e órgãos moles, enquanto CT permite a identificação de fraturas, calcificações, implantes e traumas. Conclusões: Nos relatórios encontrados na pesquisa da literatura, o uso da ressonância magnética nuclear e da tomografia computadorizada em casos postmortem, nomeado pela gênese do trauma, foi encontrada correlação entre o uso da imagem e o correto diagnóstico especialista da autópsia. [Najar-Céspedes AP, Fuentes-Martínez EDJ. Uso da ressonância magnética e da tomografia computadorizada no diagnóstico pós-morte: revisão do assunto. MedUNAB 2017; 20(2): 190-200].

Palabras-chave: Diagnóstico por Imagem; Tomografia Computadorizada por Raios X; Espectroscopia de Ressonância Magnética; Autopsia; Medicina Legal.

de las cuales los suicidios junto a los accidentes de tránsito constituyen las primeras causas. Así mismo se realizaron 273,511 exámenes médico legales, en donde la violencia interpersonal e intrafamiliar aportaron el $73.5 \%$ de los casos (3).

En este amplio conjunto, ante la naturaleza heterogénea y casuista de la actividad, para llevar a cabo el peritaje(1) del que se habla, el perito debe hacer el mejor uso de las herramientas técnicas y tecnológicas de las cuales disponga; por ejemplo, en la práctica de necropsias, apoyando sus conclusiones periciales en la información dada por las imágenes diagnósticas del cuerpo objeto de estudio(4-6).

Ahora bien, es bueno mencionar que el uso de herramientas tecnológicas en el diagnóstico clínico no reviste confusión, por el contrario, cada avance tecnológico ha traído consigo la necesidad de desarrollos nativos en el campo de la medicina. Para el caso particular de la imagenología, desde 1895 se documenta el estudio y utilización de la radiología como apoyo a la medicina forense; donde, como es de esperar, en la medida de las tecnologías emergentes, éstas se han estudiado, ensayado e incorporando en el campo $(7,8)$.

Al respecto, los estudios y utilización clínica de las imágenes producidas no son exclusividad de un único 
método (Rayos X, resonancia, etc.), ni de la medicina forense; hecho que se reafirma con las aplicaciones médicas de la resonancia magnética (RM), en 1980, época en la cual se desarrollaron numerosos estudios e investigaciones, de las cuales se rescata el reconocimiento de células de crecimiento anormal (tumores), realizada exclusivamente con el uso de la RM(7-9).

Por supuesto, estas herramientas son tan potentes que trascienden el equipamiento para la jurisprudencia médica, y es una realidad que los avances en imagenología, en especial en RM, han hecho que esta sea empleada en todo tipo de actividades y su uso postmortem haya venido en aumento; siendo soporte en aquellas autopsias donde (por religión u otras) no se permite la práctica de exámenes invasivos, captando imágenes de diferentes órganos del cuerpo, ilustrando su utilidad para emitir un correcto diagnóstico pericial de la causa de muerte(9-12).

En esta dinámica de incorporar avances tecnológicos a la actividad pericial, en la práctica de necropsias, se generó el proyecto Virtopsy ${ }^{\circledR}(11,13)$, cuya propuesta ofrece un modo no invasivo de examinar cadáveres, haciendo uso de estudios de imagenología entre los cuales se encuentra la Tomografía Computarizada (TC). Esta última tecnología, capaz de realizar diversos procesos de análisis de imagen, como reconstrucciones en volumen o mediciones morfométricas, ha logrado gran aceptación de las instituciones especializadas, debido a su rapidez en el diagnóstico y al fácil sistema de archivo y registro, permitiendo el transporte de datos recabados a especialistas apartados entre sí, sin manipulación de las muestras.

Esas mismas utilidades han llevado a popularizar el uso de la $\mathrm{RM} / \mathrm{TC}$, perfilándose como las metodologías principales para la realización de exámenes postmortem(14).

Por su parte, para Colombia durante el 2017 no se contó con investigaciones ni documentación sobre la aplicación de estas técnicas de imagenología en estudios postmortem, consecuencia lógica, puesto que dichas tecnologías conllevan grandes costos de implementación, tanto que, incluso en el llamado "primer mundo", no todos los hospitales de amplia superficie poseen su propia TC, menos aún, una unidad de RM (15). Bajo esta realidad, se prioriza destinar los recursos tanto económicos como operativos, principalmente a la atención en salud de los ciudadanos que sobreviven, y no, para el eventual uso en la administración de justicia.

Sobrellevando esta realidad, el país no puede quedar relegado en los avances tecnológicos que se están dando y dejar de lado los principios de justicia, promoviendo la impunidad en casos que por su particularidad requieran el uso de técnicas eficientes para esclarecer sus móviles. De allí la necesidad de realizar una documentación de casos en los cuales se aplicaron dichas técnicas en estudios postmortem, para que con base a estos registros se logre su posterior aplicación. Con esta intención, el presente trabajo de revisión tiene como fin analizar el uso de la RM y la TC en el diagnóstico postmortem, buscando generar inquietud en profesionales en compañía de estudiantes de las ciencias de la salud, e incitando una conciencia investigativa que se espera llegué a promover investigaciones posteriores para una futura aplicación de las técnicas y sus tecnologías asociadas en el territorio colombiano.

\section{Imagenología Postmortem}

Los estudios postmortem son todos aquellos estudios internos y externos que se practican a un cadáver para determinar la causa de muerte (16). Dentro de ellos se encuentra la necropsia, la cual es el procedimiento que estudia las alteraciones morfológicas de los órganos y tejidos como consecuencia de enfermedad o causa alguna de muerte $(17,18)$.

Bajo la definición de necropsia, la RM y la TC, junto a la autopsia convencional, son herramientas cuya función es precisar el diagnóstico, posibilitando a su vez, el estudio de hallazgos traumatológicos o patológicos, e incluso de reacciones vitales ante-mortem, siendo particularmente útil en aquellos casos de dudosa criminalidad; las imágenes demuestran con nitidez y tamaño apropiado cualquier estructura corporal, lo que significa menor invasión al cuerpo objeto de estudio $(8,13,17)$.

La principal importancia del uso de cualquiera de estas técnicas para los estudios postmortem son las facilidades que una y otra proporcionan, entregando datos fidedignos y tridimensionales de las estructuras corporales $(17,19)$, guiando la obtención de muestras biológicas, ofreciendo además la posibilidad de realizar una base de datos para futuras investigaciones entre otros especialistas, dada la capacidad de almacenamiento de la información(12).

Como complemento, las técnicas de imagenología pueden llegar a evitar procedimientos innecesarios, así como podrían llegar a reducir el número de autopsias (procedimientos invasivos), los cuales alteran el cuerpo y dificultan inspecciones posteriores $(17,20)$. En las ciencias médicas, no es de olvidar el sentido humano, para que los parientes puedan ver el cadáver incluso después de la autopsia, la cara no es sometida a disección. Con la imagen forense, los tejidos blandos y el esqueleto de la cara pueden ser examinados de manera no destructiva. Si hay necesidad de tomar muestras o fluidos del cadáver, estas podrían hacerse de forma guiada con las técnicas imagenológicas mencionadas, ofreciendo así una menor manipulación del cuerpo por parte de los patólogos y técnicos, y reduciendo el riesgo de infección para este personal.

Otra utilidad de estas técnicas se da cuando por motivos culturales, religiosos o personales, los familiares no permiten una autopsia, pero dicho examen puede ser clave 
para descubrir las causas de deceso, y de ser el caso, impartir justicia. Otra utilidad de la imagenología postmortem es la posibilidad de reevaluar un caso por un experto de una subespecialidad específica en cualquier lugar, en cualquier momento.

\section{Resonancia magnética}

La resonancia magnética $(\mathrm{RM})$ trabaja basada en las propiedades mecánico-cuánticas de los núcleos atómicos (21). Para llevar a cabo su función, hace uso de un juego de poderosos electroimanes, creando campos magnéticos al circular la corriente por las bobinas de los mismos; estos campos estimulan los átomos de hidrógeno, que, una vez excitados, vuelven a su posición de equilibrio, produciendo una señal que es recibida por los transductores, luego transformada a una señal eléctrica y posteriormente convertida en la imagen física de la estructura a estudiar(22, 23).

De la imagen resultante se desprenden múltiples posibilidades de edición, pudiendo emplear secuencias para suprimir, recortar, aumentar o resaltar imágenes. Por ejemplo, utilizar los contrastes para retirar (de la imagen) el tejido graso y aumentar la intensidad del tejido conectivo para observar en una secuencia especializada la articulación de la rodilla, o suprimir la señal del agua para aumentar la señal de tejido y observar el parénquima cerebral; la gran ventaja de la RM es que cada secuencia tiene tanto un fin determinado como una estructura, para así observar desde diferentes vistas de esquema la estructura, concluyendo en el diagnóstico más acertado(22-24).

Esta técnica posee la ventaja de poder representar lesiones de tejidos blandos y patologías claramente. Por otra parte, su uso no se limita, puede ser usada para examinar víctimas vivas de actos violentos, dado que es un método no ionizante (11). No obstante, sus prestaciones no se masifican principalmente a sus altos costos, la necesidad de escáneres de alto campo para autopsia perinatal, el tiempo prolongado entre exámenes, la imposibilidad de mostrar en forma directa el calcio en el tejido óseo, así como un altísimo riesgo de incidentes o accidentes si no se cumplen las normas de seguridad en el área de imagenología (ejemplo: Efecto misil, introducción inadvertida de pacientes con contraindicaciones, quemaduras $)(8,25)$.

\section{Tomografía computarizada}

La TC es una técnica que utiliza la combinación de Rayos $\mathrm{X}$ y sistemas informáticos para conseguir una serie de imágenes transversales de una estructura, que ofrecen información de anatomía en tres dimensiones. Gracias a ello es posible estudiar con precisión, detalles de las vísceras, huesos, tejido muscular, graso, entre otras estructuras (26, 27).
El equipo de tomografía computarizada está constituido por un gantry o cuerpo vertical, que presenta un orificio central, el cual contiene un tubo de Rayos X que gira alrededor del paciente, una mesa o camilla de exploración, donde se coloca la estructura a estudiar, el gantry emite radiaciones que pasan a través del organismo u objeto, las cuales son modificadas según el coeficiente de absorción de los diferentes tejidos. Estas radiaciones modificadas son captadas en el lado opuesto por unos detectores de estado sólido o gaseoso. La ionización producida por los Rayos X en las moléculas del detector se convierten en señales eléctricas que son transmitidas a un computador, donde se transforma en imágenes digitalizadas a la interfaz del operador(28).

Como ventajas se puede mencionar que la técnica puede detectar y representar cuerpos extraños, fracturas, gas, acumulaciones de fluidos y calcificaciones en grandes vasos. Así mismo, su uso a través de la angiografía postmortem permite la detección de rupturas u oclusiones de vasos sanguíneos con menor calibre (29). Adicionalmente, la $\mathrm{TC}$ requiere pocos minutos para su realización y esta característica permitirá que, un gran número de cadáveres puedan ser analizados (30). Sin embargo, tiene limitaciones con respecto a la evaluación de tejidos blandos y órganos internos, dado que las densidades de los tejidos ante un daño o patología pueden presentar cambios tan pequeños que no pueden ser detectados por la técnica (ejemplo: Para el caso de una hemorragia subaracnoidea, no se podría conocer si la causa fue por aneurisma o por malformación arteriovenosa).

\section{Metodología}

Se realizó una búsqueda de información en las bases de datos PubMed, Science Direct, Springer Journal y en el motor de búsqueda Google Académico, usando los términos MESH: "Computed Tomography", "Magnetic Resonance", "Autopsy", "Postmortem" y "Forensic Medicine", publicada en el periodo 2008/01/01$2015 / 12 / 31$, filtrándose la información por tipo de artículo en donde se seleccionaron los reportes de caso, artículos originales de investigación y revisiones, publicadas en inglés. Después se realizó un análisis de los títulos, resúmenes y lectura de los artículos disponibles en su versión completa, para posteriormente seleccionarse 50 . Adicionalmente, se añadieron otros documentos que ampliarán la información para algunos temas, con base en las referencias bibliográficas o en la búsqueda en PubMed.

El estudio de casos, como metodología de investigación, es el estudio de la particularidad y de la complejidad de un caso singular. Se hace uso de este método cuando se tiene un interés especial en el caso en sí mismo, para llegar a comprender su actividad en circunstancias importantes, buscando siempre en detalle la interacción con sus contextos (31). En este marco metodológico se desprenden los siguientes casos: 


\section{A. Fractura de la bóveda craneal}

La RM, por mérito propio, es la mejor técnica para observar el sistema nervioso central. Ofrece imágenes únicas de todo el contorno del encéfalo; y a su vez, haciendo uso de las múltiples secuencias de contraste, permite realzar la protuberancia, el líquido cefalorraquídeo y la totalidad de la morfo fisiología cerebral(32-34).

Gracias a la RM se observa en detalle efluvios y sangrados en regiones centralizadas, como el hipocampo, el cuerpo calloso y los plexos coroideos; sirviendo también en la identificación de traumas junto a hemorragias subdurales y subaracnoideos, así como en probables daños en el sistema ventricular (35). Por lo anterior es la herramienta idónea a usar en pacientes que han fallecido por poli-traumatismos en accidente de tránsito, por golpes con objetos contundentes o caídas desde grandes alturas, también, claro está, para observar la complejidad del daño y los factores que causaron su muerte(36) (Figura 1).
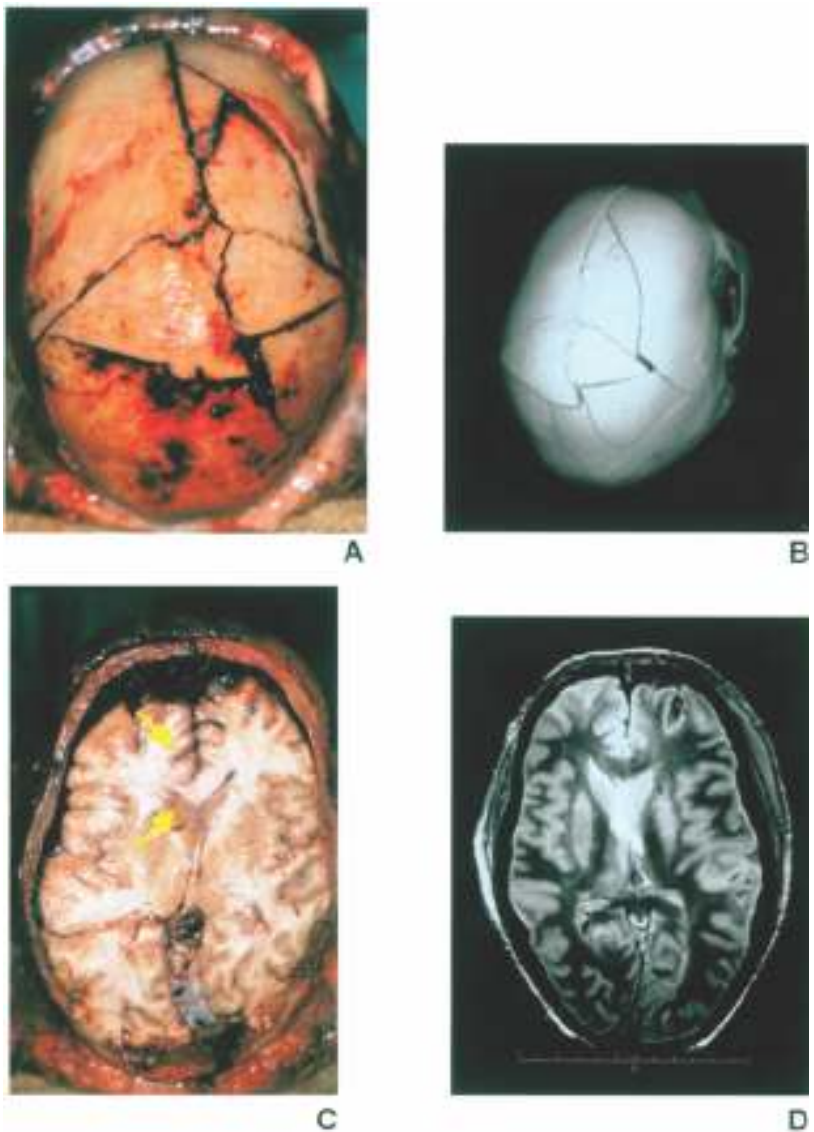

Fuente: Tomado de Thali MJ y cols. J Forensic Sci. 2003; 48(2):386403.

Figura 1. Fractura de la bóveda craneal de paciente de 27 años por caída de un árbol.

(a) Imagen de la autopsia, (b) Reconstrucción tridimensional de la tabla ósea, (c) Corte axial de la autopsia, (d) Corte axial en $\mathrm{T} 2$ resonancia magnética por imagen.
No sobra recalcar, que esta técnica no sustituye del todo la autopsia convencional (Figura 1a), pero sí, es una poderosa herramienta para visualizar elementos omitidos por el estudio humano; permitiendo reconstrucciones tridimensionales o cortes axiales, donde sea fácilmente observable la posición y el tamaño de la lesión (Figura 1b y 1d); mejora además la comprensión de masas circunscritas de la cavidad que la contiene normalmente, por un orificio natural o accidental (flechas amarillas en la Figura 1c), lo que equivale a determinar magnitudes físicas, como distancias, tipo de objetos, entre otros.

\section{B. Herida por arma cortopunzante en región torácica}

Las heridas por arma de fuego, arma cortopunzante y los traumas por accidentes constituyen un elevado desafío para el médico patólogo encargado de la necropsia, puesto que se ocasionan lesiones en diversos tejidos y órganos, que ensombrecen el diagnóstico.

Para Jackowski y colaboradores(37), un caso crítico para el diagnóstico hace su aparición en aquellas heridas presentes en la región torácica, dado que en esta región se ubican diferentes estructuras esenciales para la vida humana, cualquier omisión puede conducir al indeseado mal diagnóstico, o no menos grave, a establecer causas idiopáticas de muerte(38).

Esta situación contradice a la autopsia convencional, obligando a buscar alternativas; con la RM puede apreciarse cardiopatías complejas (ejemplo: Insuficiencia cardiaca, endocarditis, perforaciones, contusiones mediastinales y cardiacas) $(39,40)$; y a nivel respiratorio, cualquier tipo de lesión traumática que provoque colapso pulmonar, como lo son aplastamientos, asfixia mecánica, traumas cerrados de tórax, entre otros $(40,41)$. Igualmente, haciendo uso de ella se pueden diagnosticar tromboembolismos, derrames pleurales, procesos infecciosos (neumonías, tuberculosis), patologías por trauma, neoplasias, hemotórax, neumotórax y quilotórax (42), enfermedades crónicas como tumores mediastinales pleurales, pulmonares y los enfisemas con consolidaciones a nivel pulmonar. Contrastando las imágenes por resonancia se puede apreciar en todo contexto el parénquima pulmonar y así encontrar el diagnóstico acertado $(43,44)$.

La imagen detallada obtenida por RM, facilita observar la completa trayectoria de lesiones y los daños adyacentes en su recorrido (Figura 2c), en la cual se muestra la lesión en el tejido cutáneo, apoyada a una TC para realizar la posterior ruta de la lesión $(44,45)$. Como se dijo, el contraste es una ventaja de la RM (Figura 2b) afirmando la lesión del tejido miocárdico de la autopsia (Figura 2a) con aparición de coágulo intracardial; y a su vez se observa lesión a nivel vascular (Figura 2d). 

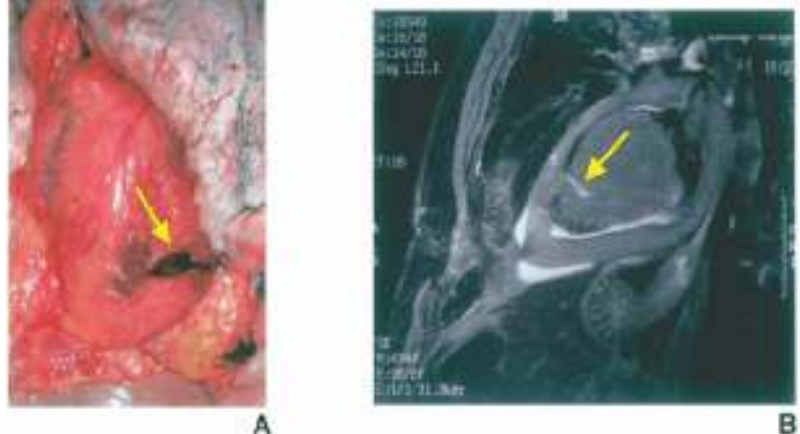

A
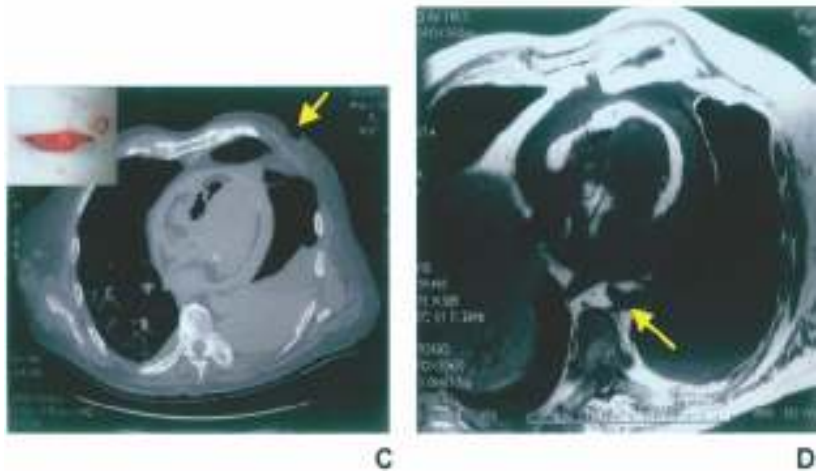

Fuente: Tomado de Thali MJ y cols. J Forensic Sci. 2003;48 (2):386-403.

Figura 2. Paciente quien fallece por herida cortopunzante localizada en región torácica. (a) Imagen de la autopsia, (b) Corte coronal RM, (c) Tomografía computarizada y resonancia magnética por imagen, (d) Corte axial resonancia magnética.

\section{Traumas provocados por accidentes de tránsito}

El sistema osteomuscular (compuesto básicamente por huesos, tendones y tejido muscular) además de ser responsable de la adecuada locomoción, sirve de armazón y sostén mecánico para cada una de las estructuras internas. La aplicación de la RM en la evaluación de este sistema ayuda a identificar de manera clara cualquier tipo de lesión, sabiendo así el tipo y la magnitud del daño causado (21). Su importancia es tal que, la mera observación de las articulaciones, proporciona información relevante sobre el tiempo de vida de los cuerpos en estudio, así como de las patologías y medicaciones conexas que pudiesen llegar a producir deformaciones o cambios articulares. Simultáneamente, detallar los tendones, dada su propensión a sufrir rupturas por cualquier tipo de trauma contundente, puede verse reflejada en RM. A su turno, el tejido muscular una vez afectado, presenta en la RM, hematomas, laceraciones y otros daños (Figura 3) que, al llevar a cabo la autopsia, reafirman los resultados dados por la imagen $(46,47)$.

En otro estudio, se comparó los resultados de la autopsia convencional con los obtenidos utilizando TC y RM, queriendo identificar y evaluar la biomecánica del trauma
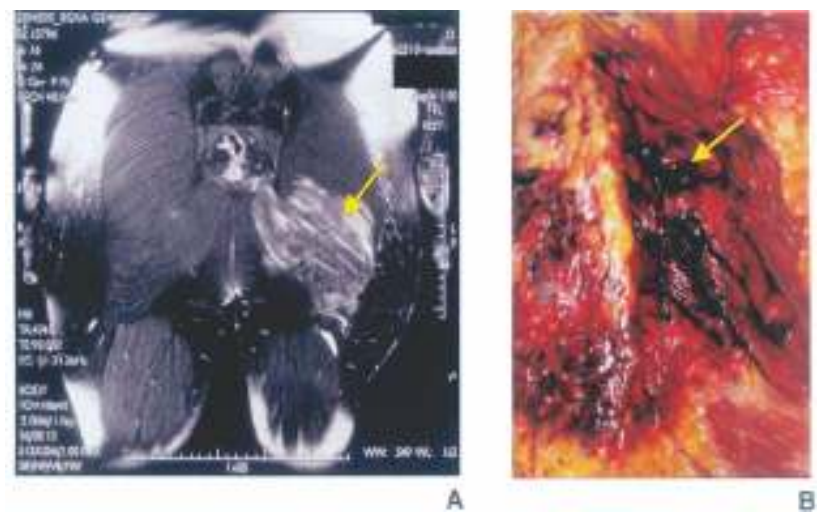

Fuente: Tomado de Thali MJ y cols. J Forensic Sci. 2003; 48(2):386-403.

Figura 3. Paciente fallecido por trauma causado por arrollamiento vehicular. (a) Corte coronal resonancia magnética, (b) Imagen de la autopsia.

en las causas de muerte (48). En uno de los individuos de estudio se notó, a partir de la reconstrucción en $3 \mathrm{D}$ por TC, la fractura de la apófisis odontoide (Figura 4a) y por su parte, con la RM se apreció el desplazamiento de medula espinal causado por la fractura (Figura 4b). En estudios semejantes, se investigó la efectividad del diagnóstico usando TC postmortem con víctimas de accidentes de tránsito, las cuales presentaron lesiones en cabeza, cuello, tórax, abdomen y pelvis, encontrándose que la TC postmortem puede detectar o presumir un diagnóstico de trauma fatal en el momento de determinar la causa de muerte $(49,50)$.
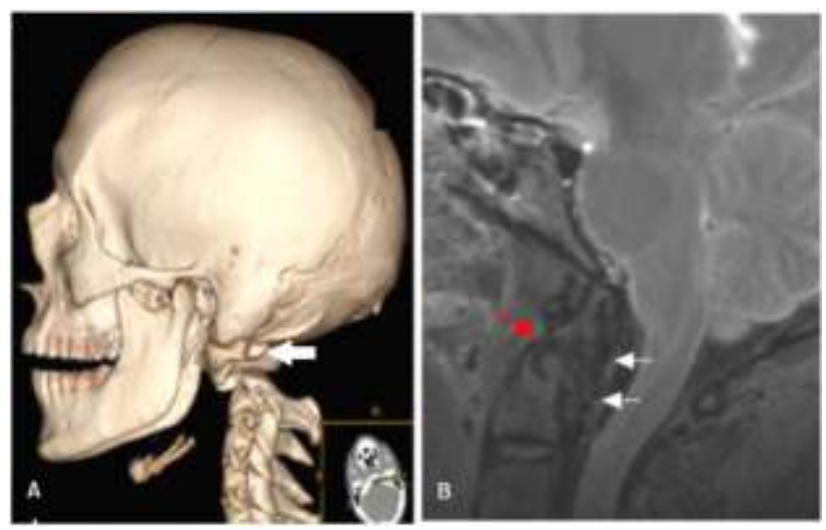

Fuente: Modificado de Yen K y cols. Int J Legal Med. 2005; 119(3):129-36.

Figura 4. Paciente fallecido por trauma causado por arrollamiento vehicular. (a) Tomografía computarizada, (b) Resonancia magnética.

\section{D.Ahogamiento}

El sistema gastrointestinal se encuentra rodeado de órganos sólidos, hígado y páncreas, y huecos como el estómago y los intestinos; irrigados por vasos que van desde los más 
grandes: Arteria aorta, vena cava cruzando todo el sistema digestivo, hasta los más pequeños, pero no menos complejos vasos sanguíneos $(46,51,52)$.

Las imágenes por RM de este sistema aportan datos para el hallazgo de patologías de tipo traumático (laceraciones, fisuras y roturas de órganos huecos), ocasionadas, ya sea por heridas por arma de fuego, heridas por arma cortopunzante o traumas contundentes (caídas de gran altura y golpes) (53, 54), así como de tipo accidental como el ahogamiento (Figura 5); en donde, haciendo uso de los potenciadores, se muestra la abundante cantidad de líquido con partes solidas en estómago y duodeno (Figura 5b) para luego realizar la autopsia (Figura 5a) donde se ve pinzamiento de la primera porción del duodeno (flechas).
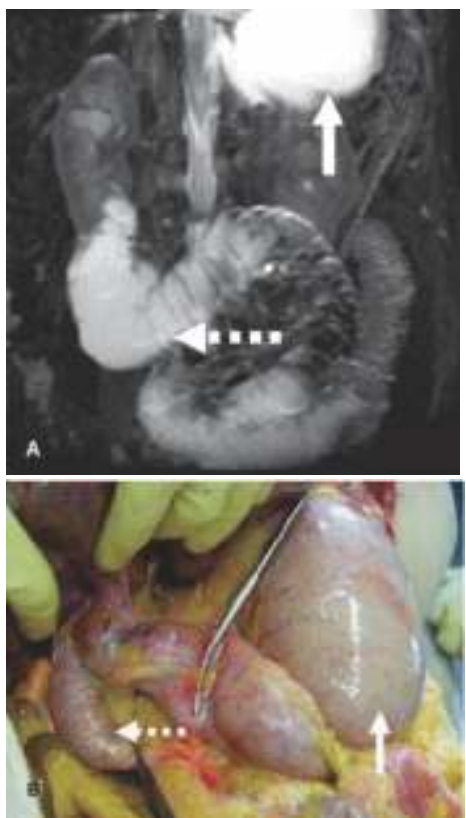

Fuente: Tomado de Dirnhofer R y cols. Radiographics. 2006; 26(5):1305-33.

Figura 5. Resonancia magnética y autopsia a individuo fallecido por ahogamiento. (a) Corte coronal resonancia magnética, (b) Imagen de la autopsia.

\section{E. Gas intrahepático}

La presencia de Gas Intrahepático (GIH), es un hallazgo frecuente en la TC postmortem en casos de muerte no traumática (55-57). Por ello, se han realizado investigaciones sobre su detección por $\mathrm{TC}$, para posteriormente confirmar mediante la autopsia convencional. En uno de los reportes, se evaluó la ubicación y ocurrencia de GIH, mediante imágenes por TC multicorte (TCM) en 208 personas fallecidas no traumáticamente(56). La ubicación de GIH fue clasificado en una escala de 0 a 3 , siendo 0 la no aparición de ningún gas; 1 , gas sólo en el lóbulo izquierdo (Figura 6a); 2, gas en el lóbulo izquierdo y el segmento anterior del lóbulo derecho (Figura 6b); y 3, gas en el lóbulo izquierdo con los segmentos anterior y posterior del lóbulo derecho (Figura 6c), encontrándose que la mayoría de los casos poseían este hallazgo en el tipo 3 de la escala y que esto se correlacionaba con la aparición de distensión intestinal, y el periodo transcurrido entre el paro cardiorespiratorio y el posterior examen y la autopsia.

\section{F. Muerte por patología preexistente}

En el caso de patologías preexistentes, se estudió el caso de una mujer de 77 años que murió repentinamente después de someterse a una operación de aneurisma cerebral. La TC postmortem de cráneo mostro que la hemorragia propia de la cirugía desapareció sin ninguna complicación, pero la tomografía de tórax mostró múltiples tumores pulmonares metastásicos con masas anormales en los bronquios (Figura 7a) y derrame pleural (Figura 7b), dados estos hallazgos se estima que el fallecimiento ocurrió por asfixia causada mediante metástasis pulmonar y tumores endobronquiales. Aunque una autopsia tradicional no se realizó, la TC postmortem proporcionó una fuerte evidencia para la detección de la causa de muerte, evitando un riesgo judicial en la gestión del hospital involucrado(58).
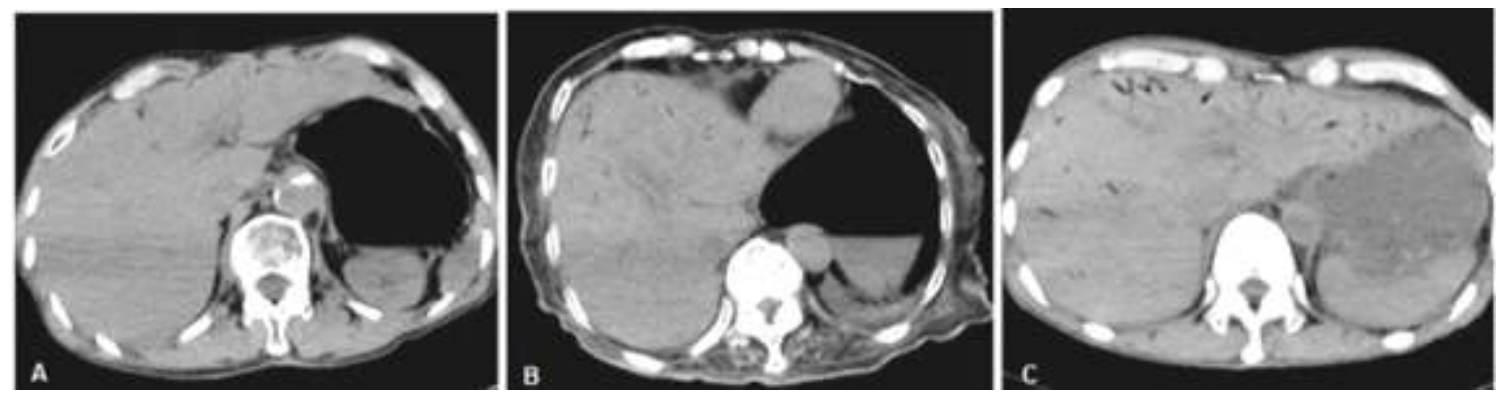

Fuente: Tomado de Takahashi N y cols. Jpn J Radiol. 2009; 27(7):264-8.

Figura 6. Tomografía computarizada multicorte de abdomen, gas intrahepático en el lóbulo izquierdo. (a) Tipo 1 , (b) Tipo 2, (c) Tipo 3. 

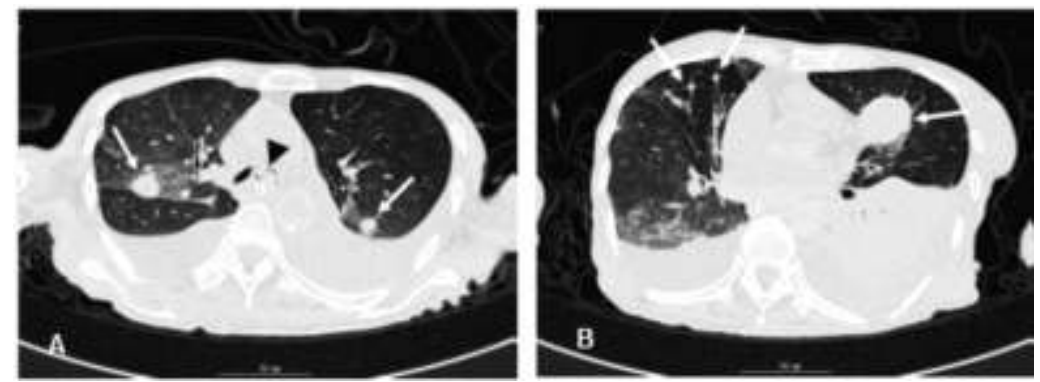

Fuente: Tomado de Takahashi N y cols. Jpn J Radiol. 2009; 27(8):316-9.

Figura 7. Tomografía computarizada de tórax, paciente de 77 fallecido por metástasis pulmonar. (a) Evidencia de masa anormal endobronquial, (b) Evidencia de derrame pleural bilateral.

\section{G. Identificación de cadáveres NN (No Name)}

La TC también ha sido usada para la identificación de sujetos, ya que esta puede detectar características propias de los cuerpos, tales como son implantes, cirugías o fracturas antiguas (59). Puede verse, por ejemplo, para los estudios realizados por Dedouit y colaboradores, a 35 cuerpos; de las 35 exploraciones, diez revelaron malformaciones esqueléticas, fracturas antiguas, o fracturas no consolidada en el cuello de fémur derecho (Figura 8a); una fractura de la primera vértebra cervical a nivel pedicular izquierdo (Figura 8b); una columna lumbar con material de osteosíntesis (Figura 8c); una calcificación en el ligamento tirohioideo izquierdo (Figura 8d), espondilolistesis bilateral ístmica de la quinta vértebra lumbar, y un angioma vertebral torácico $(60,61)$.

Algunas anomalías esqueléticas fueron confirmadas por las investigaciones de la policía: El fallecido quien tuvo el material de osteosíntesis tenía una historia pasada de la fractura de la columna y la cirugía, el sujeto que presento fractura de vértebra cervical pedicular izquierda tenía un historial reciente de fractura cervical. A excepción de la prueba del material de osteosíntesis, ninguno de los

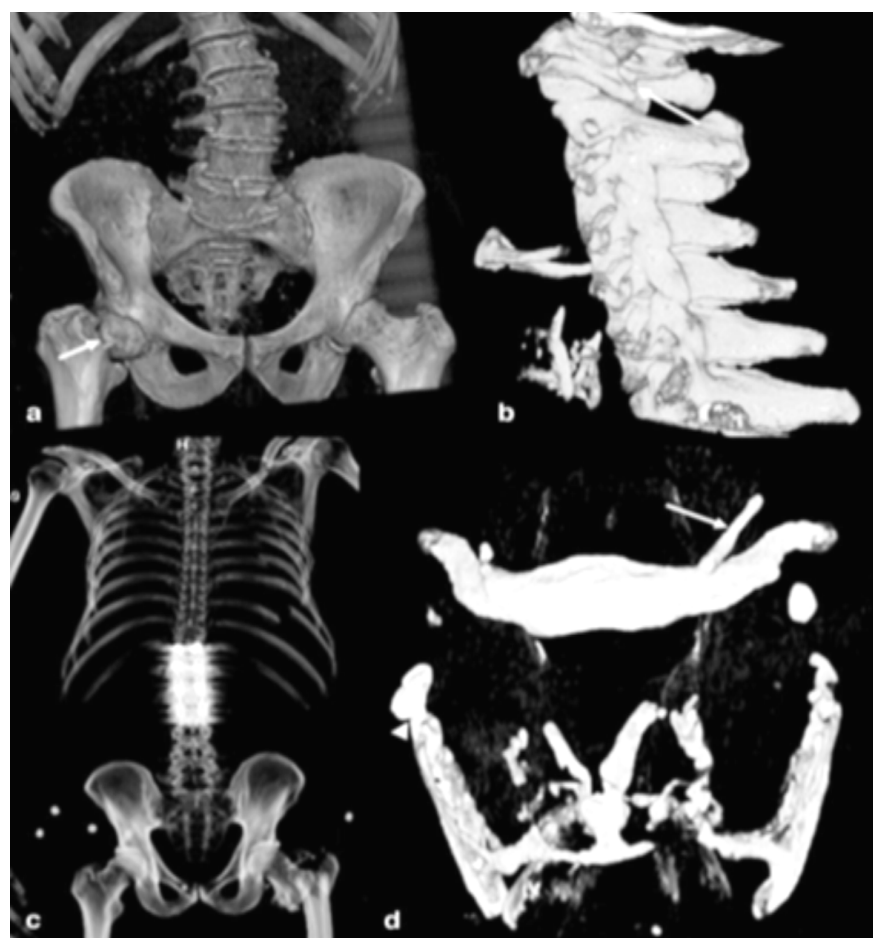

Fuente: Tomado de Dedouit F y cols. Int J Legal Med. 2007; 121(6):507-10.

Figura 8. Tomografía computarizada de posibles malformaciones esqueléticas. (a) Fractura no consolidada en el cuello del fémur, (b) Fractura de primera vértebra cervical, (c) Columna lumbar con material de osteosíntesis, (d)Angioma vertebral torácico. 
hallazgos mencionados se ve durante la autopsia convencional $(60)$.

\section{Conclusiones}

La imagenología postmortem no pretende desplazar, ni ser un sustituto de la autopsia convencional, de hecho, estas técnicas pueden aumentar, el número de éstas o cambiar su enfoque, tanto médico como legal, ampliando el espectro de la autopsia convencional, posibilitando a su vez el acervo de archivo de imágenes para posteriores investigaciones forenses.

Con lo anterior en mente, es bueno resaltar los beneficios para la investigación en los estudios forenses de la RM, entre ellos, se cuenta con una mejor definición en la imagen, capacidad de reducir o intensificar la señal de cualquier tipo de tejido, posibilidad de realizar reconstrucciones en 3D para realizar esquemas completos de cualquier región del cuerpo, entre otros.

Por su parte, la TC proporciona distintos grados de libertad para la imagen, facilitando establecer una correlación directa con los datos radiológicos ya adquiridos, siendo útil cuando el individuo en estudio presenta artefactos en su cuerpo. Como la RM, el uso de la TC postmortem, puede detectar o presumir trauma fatal en el diagnóstico de la causa de muerte después de accidentes u otros de conclusiones fatales. Tanto es así que, combinado con una angiografía, se analizan con mayor facilidad las lesiones vasculares periféricas en traumas, ya sean de naturaleza cerrada o abierta.

Desde luego, y a pesar del buen recibo de sus sobresalientes ventajas, la imagenología postmortem carece de otras características diagnósticas como color, textura u olor; las cuales pueden llegar a ser relevantes, y no estarían presentes en las imágenes.

Otro aspecto a considerar es como las interpretaciones de los hallazgos encontrados en las imágenes van a depender de factores tales como la causa de muerte, cambios normales postmortem, efectos de la resucitación cardiopulmonar, entre otros. Por lo tanto, los radiólogos clínicos pueden confundirse o malinterpretar hallazgos a partir de la radiología en cadáveres, y esto obliga a notar que la radiología forense no es la misma radiología clínica aplicada.

Finalmente, existe otra dimensión no menos importante, referente a las cuestiones pecuniarias, dado que no se recomienda generar sinergias en equipos hospitalarios, pese a poder usar los mismos tomógrafos o resonadores (que sirven para los pacientes en general) en labores forenses, este hecho podría atentar contra la seguridad y sanidad de personas e instalaciones; quedando en discusión la necesidad de conformación de unidades de imágenes diagnosticas forenses.

\section{Conflicto de intereses}

Los autores declaran no tener conflicto de interés.

\section{Referencias}

1. Caballero $\mathrm{C}$, Moreno $\mathrm{H}$. Tratado de medicina legal: Juristas y medicina. 3ra ed. Bucaramanga, Colombia: Editorial UNAB; 1996.

2. Butchart A, Mikton C, Dahlberg LL, Krug EG. Global status report on violence prevention 2014. Inj Prev. 2015;21(3):213.

3. Legal INdM, Violencia GCdRNs. Forensis 2015. Datos para la vida. . 1 ed. Colombia2016. p. 729.

4. Payne-James JJ, Stark MM. Clinical Forensic Medicine: History and Development. In: Stark MM, editor. Clinical Forensic Medicine: A Physician's Guide. Totowa, NJ: Humana Press; 2011. p. 1-44.

5. Ludwig J. Postmortem Imaging Techniques. In: Waters BL, editor. Handbook of Autopsy Practice. Totowa, NJ: Humana Press; 2009. p. 99-103.

6. Medical-Legal Aspects. Radiological Reporting in Clinical Practice. Milano: Springer Milan; 2008. p. 27-38.

7. Arce Mateos FP, Fernández Fernández FÁ, Mayorga Fernández MM, Gómez Román J, Val Bernal JF. La autopsia clínica. 2009;7(1):10.

8. Flach PM, Thali MJ, Germerott T. Times have changed! Forensic radiology--a new challenge for radiology and forensic pathology. AJR Am J Roentgenol. 2014;202(4):W325-34.

9. Common Sense in Clinical and Preclinical Diagnosis. Radiological Reporting in Clinical Practice. Milano: Springer Milan; 2008. p. 79-80.

10. Thali MJ, Jackowski C, Oesterhelweg L, Ross SG, Dirnhofer R. VIRTOPSY - the Swiss virtual autopsy approach. Leg Med (Tokyo). 2007;9(2):100-4.

11. Tejaswi KB, Hari Periya EA. Virtopsy (virtual autopsy): A new phase in forensic investigation. J Forensic Dent Sci. 2013;5(2):146-8.

12. Thayyil S, Chandrasekaran M, Chitty LS, Wade A, Skordis-Worrall J, Bennett-Britton I, et al. Diagnostic accuracy of post-mortem magnetic resonance imaging in fetuses, children and adults: a systematic review. Eur J Radiol. 2010;75(1):e142-8.

13. Thali MJ, Yen K, Schweitzer W, Vock $P$, Boesch $C$, Ozdoba C, et al. Virtopsy, a new imaging horizon in forensic pathology: Virtual autopsy by postmortem multislice computed tomography (MSCT) and magnetic resonance imaging (MRI) - a feasibility study. Journal of Forensic Sciences. 2003;48(2):386-403.

14. Stawicki SP, Aggrawal A, Dean AJ, Bahner DA, Steinberg SM, Stehly CD, et al. Postmortem use of advanced imaging techniques:Is autopsy going digital? OPUS 12 Scientist. 2008;2(4):17-26.

15. Investor's Business D. MRI prices all over the map. Investors Business Daily. 2015:A02.

16. Levy AD. Postmortem Radiology and Imaging Postmortem Radiology and Imaging 2012 [Available from: http://emedicine.medscape.com/ article/1785023overview.

17. Vogel H, Houck JASJSM. Postmortem Imaging. Encyclopedia of Forensic Sciences. Waltham: Academic Press; 2013. p. 243-60. 
18. Waters BL. Ensuring Quality in the Hospital Autopsy. In: Waters BL, editor. Handbook of Autopsy Practice. Totowa, NJ: Humana Press; 2009. p. 3-9.

19. Lequin MH, Huisman TAGM. Postmortem MR Imaging in the Fetal and Neonatal Period. Magnetic Resonance Imaging Clinics of North America. 2012;20(1):129-43.

20. Koch V. Methodenvergleich zwischen postmortaler CT und MRT Bildgebung in der forensischen Begutachtung: Graz Medical University; 2009.

21. Jeffery AJ. The role of computed tomography in adult post-mortem examinations: an overview. Diagnostic Histopathology. 2010;16(12):546-51.

22. Grover VP, Tognarelli JM, Crossey MM, Cox IJ, TaylorRobinson SD, McPhail MJ. Magnetic Resonance Imaging: Principles and Techniques: Lessons for Clinicians. J Clin Exp Hepatol. 2015;5(3):246-55.

23. Sederman AJ, Wang M. 4 - Magnetic resonance imaging. Industrial Tomography: Woodhead Publishing; 2015. p. 109-33.

24. Nitz WR, Balzer T, Grosu DS, Allkemper T. Principles of Magnetic Resonance. In: Reimer P, Parizel PM, Meaney JFM, Stichnoth FA, editors. Clinical MR Imaging. Berlin, Heidelberg: Springer Berlin Heidelberg; 2010. p. 1-105.

25. Thayyil S, Chitty LS, Robertson NJ, Taylor AM, Sebire NJ. Minimally invasive fetal postmortem examination using magnetic resonance imaging and computerised tomography: current evidence and practical issues. Prenat Diagn. 2010;30(8):713-8.

26. Panda A, Kumar A, Gamanagatti S, Mishra B. Virtopsy Computed Tomography in Trauma: Normal Postmortem Changes and Pathologic Spectrum of Findings. Curr Probl Diagn Radiol. 2015;44(5):391-406.

27. Cierniak R. Technical Concepts of X-ray Computed Tomography Scanners. X-Ray Computed Tomography in Biomedical Engineering. London: Springer London; 2011. p. 21-62.

28. De la Cerda Romero A. Equipos de Tomografia Computerizada (TAC). Revista Digital para Profesionales de la Ensenanza. 2009; 5.

29. Busardo F, Frati P, Guglielmi G, Grilli G, Pinto A, Rotondo $A$, et al. Postmortem-computed tomography and postmortem-computed tomography-angiography: a focused update. Radiologia Medica. 2015;120(9):810-23.

30. Brough A, Morgan B, Rutty G. Postmortem computed tomography (PMCT) and disaster victim identification. Radiologia Medica. 2015;120(9):866-73.

31. Stake RE. Investigación con estudio de casos. 2000.

32. Thayyil S, De Vita E, Sebire NJ, Bainbridge A, Thomas D, Gunny R, et al. Post-mortem cerebral magnetic resonance imaging T1 and T2 in fetuses, newborns and infants. European Journal of Radiology. 2012;81(3): e232-e8.

33. Zhang Z, Liu S, Lin X, Sun B, Yu T, Geng H. Development of fetal cerebral cortex: assessment of the folding conditions with post-mortem Magnetic Resonance Imaging. International Journal of Developmental Neuroscience. 2010;28(6):537-43.

34. Pedraza S. [Usefulness of magnetic resonance imaging in traumatic brain injury]. Med Intensiva. 2013;37(6):373-4

35. Yushkevich PA, Avants BB, Pluta J, Das S, Minkoff D, Mechanic-Hamilton $D$, et al. A high-resolution computational atlas of the human hippocampus from postmortem magnetic resonance imaging at $9.4 \mathrm{~T}$. Neuroimage. 2009;44(2):385-98.

36. Colleran GC, Moynagh MR, Tavernaraki K, Shelly MJ, Eustace SJ, Kavanagh EC. Whole-body magnetic resonance imaging: emerging applications. Semin Musculoskelet Radiol. 2010;14(1):57-67.

37. Jackowski C, Christe A, Sonnenschein M, Aghayev E, Thali MJ. Postmortem unenhanced magnetic resonance imaging of myocardial infarction in correlation to histological infarction age characterization. European Heart Journal. 2006;27(20):2459-67.

38. Bisset RAL, Thomas NB, Turnbull IW, Lee $S$. Postmortem examinations using magnetic resonance imaging: four year review of a working service. British Medical Journal. 2002;324(7351):1423-4.

39. Jackowski C, Warntjes MJB, Berge J, Bar W, Persson A. Magnetic resonance imaging goes postmortem: noninvasive detection and assessment of myocardial infarction by postmortem MRI. European Radiology. 2011;21(1):70-8.

40. Ruder TD, Paula P, Hatch GM, Ampanozi G, Thali MJ, Schweitzer W. Science into practice: Post-mortem imaging provides conclusive evidence in a nonsuspicious death. Journal of Forensic Radiology and Imaging. 2014;2(2):80-4.

41. Taylor AM, Sebire NJ, Ashworth MT, Schievano S, Scott RJ, Wade A, et al. Postmortem cardiovascular magnetic resonance imaging in fetuses and children: a masked comparison study with conventional autopsy. Circulation. 2014;129(19):1937-44.

42. Rosales Uvera SG, Morelos Guzmán M, Vázquez Lamadrid J. Cor triatriatum dexter: Uso de resonancia magnética cardiovascular en su diagnóstico a propósito de un caso. Revista mexicana de cardiología. 2012;23:12-6

43. Narin B, Arman A, Arslan D, Simsek M, Narin A Assessment of cardiac masses: magnetic resonance imaging versus transthoracic echocardiography. Anadolu Kardiyol Derg. 2010;10(1):69-74.

44. Breeze AC, Jessop FA, Set PA, Whitehead AL, Cross JJ, Lomas DJ, et al. Minimally-invasive fetal autopsy using magnetic resonance imaging and percutaneous organ biopsies: clinical value and comparison to conventional autopsy. Ultrasound Obstet Gynecol. 2011;37(3):317-23.

45. Peschel O, Szeimies U, Vollmar C, Kirchhoff S. Postmortem 3-D reconstruction of skull gunshot injuries. Forensic Sci Int. 2013;233(1-3):45-50.

46. Patowary A. Virtopsy: One step forward in the field of forensic medicine. Journal of Indian Academic of Forensic Medicine. 2008;30(1):32-6.

47. Cha JG, Kim DH, Paik SH, Park JS, Park SJ, Lee HK, et al. Utility of postmortem autopsy via whole-body imaging: initial observations comparing MDCT and 3.0 T MRI findings with autopsy findings. Korean $\mathrm{J}$ Radiol. 2010;11(4):395-406.

48. Yen K, Sonnenschein M, Thali MJ, Ozdoba C, Weis J, Zwygart K, et al. Postmortem multislice computed tomography and magnetic resonance imaging of odontoid fractures, atlantoaxial distractions and ascending medullary edema. International Journal of Legal Medicine. 2005;119(3):129-36.

49. Shiotani S, Shiigai M, Ueno Y, Sakamoto N, Atake S, Kohno $M$, et al. Postmortem computed tomography findings as evidence of traffic accident-related fatal injury. Radiat Med. 2008;26(5):253-60.

50. Flach PM, Ross SG, Bolliger SA, Preiss US, Thali MJ, Spendlove D. Postmortem Whole-Body Computed Tomography Angiography Visualizing Vascular Rupture in a Case of Fatal Car Crash. Archives of Pathology \&amp; Laboratory Medicine. 2010;134(1):115-9. 
51. Filograna L, Bolliger SA, Ross SG, Ruder T, Thali MJ. Pros and cons of post-mortem CT imaging on aspiration diagnosis. Leg Med (Tokyo). 2011;13(1):16-21.

52. Sogawa N, Michiue T, Ishikawa T, Inamori-Kawamoto $O$, Oritani S, Maeda H. Postmortem CT morphometry of great vessels with regard to the cause of death for investigating terminal circulatory status in forensic autopsy. Int J Legal Med. 2015;129(3):551-8.

53. Raux C, Saval F, Rouge D, Telmon N, Dedouit F. Diagnosis of drowning using post-mortem computed tomography - state of the art. Arch Med Sadowej Kryminol. 2014;64(2):59-75.

54. Lo Re G, Vernuccio F, Galfano MC, Picone D, Milone L, La Tona G, et al. Role of virtopsy in the post-mortem diagnosis of drowning. Radiol Med. 2015;120(3):304-8.

55. Fischer F, Grimm J, Kirchhoff C, Reiser MF, Graw M, Kirchhoff S. Postmortem 24-h interval computed tomography findings on intrahepatic gas development and changes of liver parenchyma radiopacity. Forensic Sci Int. 2012;214(1-3):118-23.

56. Takahashi N, Higuchi T, Shiotani M, Maeda H, Hirose Y. Intrahepatic gas at postmortem multislice computed tomography in cases of nontraumatic death. Jpn J Radiol. 2009;27(7):264-8.
57. Jackowski C, Sonnenschein M, Thali MJ, Aghayev E, Yen $\mathrm{K}$, Dirnhofer R, et al. Intrahepatic gas at postmortem computed tomography: forensic experience as a potential guide for in vivo trauma imaging. J Trauma. 2007;62(4):979-88.

58. Takahashi N, Higuchi T, Shiotani M, Maeda H, Sasaki O. Multiple lung tumors as the cause of death in a patient with subarachnoid hemorrhage: postmortem computed tomography study. Jpn J Radiol. 2009;27(8):316-9.

59. Lorkiewicz-Muszynska D, Kociemba W, Zaba C, Labecka M, Koralewska-Kordel M, Abreu-Glowacka M, et al. The conclusive role of postmortem computed tomography (CT) of the skull and computer-assisted superimposition in identification of an unknown body. International Journal of Legal Medicine. 2013:127(3):653-60.

60. Dedouit F, Telmon N, Costagliola R, Otal P, Florence LL, Joffre $F$, et al. New identification possibilities with postmortem multislice computed tomography. Int J Legal Med. 2007;121(6):507-10.

61. Dedouit F, Savall F, Mokrane FZ, Rousseau H, Crubezy E, Rouge D, et al. Virtual anthropology and forensic identification using multidetector CT. Br J Radiol, 2014;87(1036):20130468. 